\title{
A Context-Aware Service Platform to Support Continuous Care Networks for Home-Based Assistance
}

\author{
Federica Paganelli and Dino Giuli \\ Dept. of Electronics and Telecommunications, University of Firenze \\ via S. Marta 3, \\ 50139 Firenze, Italy \\ \{federica.paganelli, dino.giuli\}@unifi.it
}

\begin{abstract}
Efficient and effective treatment of chronic disease conditions requires the implementation of emerging continuous care models. These models pose several technology-oriented challenges for home-based continuous care, requiring assistance services based on collaboration among different stakeholders: health operators, patient relatives, as well as social community members. This work describes a context-aware service platform designed for improving patient quality of life by supporting care team activity, intervention and cooperation. Leveraging on an ontology-based context management middleware, the proposed architecture exploits information coming from biomedical and environmental sensing devices and from patient social context in order to automate context-aware patient case management, especially for alarm detection and management purposes.
\end{abstract}

Keywords: care networks, home care, context model, ontology.

\section{Introduction}

At present, the growing percentage of elderly population in industrialized nations is progressively increasing the percentage of people affected by disabilities and chronic disease conditions. According to recent results of a survey funded by the European Commission [1], Europe and Japan will experience the most pronounced ageing trends up to 2050. The share of the above 60 age group will be around $37 \%$ in Europe and even more in Japan, and slightly lower in North America (27\%). As a consequence, the increase in the share of persons surviving to very old ages $(80+)$ may lead to an increase in the prevalence of chronic and degenerative diseases. The "Survey of Health, Ageing and Retirement in Europe" [1] provides a clear picture of the problem.

Advances in technologies and AmI context-aware system design are thus required in order to address two main objectives:

- improving quality of life of both patients and their closest relatives. In fact, chronic conditions cause limitations to everyday life activities, but they may dramatically decrease the quality of life of patient family members too [2]; 
- providing cost savings in long-term care services provision. Ageing and chronic conditions treatment require continuous and long-term assistance services. Such services cannot be afforded with traditional approaches, which are instead conceived for the treatment of acute health problems.

In this application scenario, services which can be provided by an AmI system have been classified into the following categories [3]:

- Emergency treatment: services for emergency detection and management

- Autonomy enhancement: services for user assistance for primary needs and/or everyday activities (nutrition, medicine taking, vital signs monitoring, etc.).

- Comfort: services allowing better life quality (e.g. education, socialization, etc.)

These services should be adaptive, easy to use, and strongly personalized according to user requirements, needs and disabilities. In most existing AmI contextaware systems, the focus is on the requirements of the person needing assistance ("patient"), whereas requirements coming from the heterogeneous community of persons which are involved in patient care and assistance are often not taken into account into system design.

On the contrary, nowadays it has been widely recognized that the care of people affected by chronic conditions requires the development of novel models, called continuous care models. These models need an organized system of care, based on the active role and cooperation of heterogeneous actors, including patient and his/her family, health care teams (e.g., general practitioners, physicians, nurses), and social community actors (for instance: volunteers and self-aid groups).

Moving from these issues, this work is being done as part of the KAMER project, which has the objective to design and realize an AmI service platform for home-based assistance of elderly and persons affected by chronic conditions. The main objective of the project is to design and develop a service platform aiming at improving patient quality of life by offering instruments and services supporting cooperating care network members. Care network members are, for instance, general practitioners, medical specialists, nurses, social operators, volunteers, and, above all, patient relatives. Even if they play different roles in the care network, they have some common requirements: for instance, they need to access patient records, they can be asked for intervention in critical situations (e.g. emergency) and they should be aware of responsibilities and actions of other care team members.

The paper is structured as follows: Section 2 introduces main aspects of novel continuous care models for treatment of chronic conditions. Section 3 discusses related work. Section 4 describes our reference application scenario and Section 5 illustrates the overall service platform architecture for Continuous Care Networks. Section 6 provides an overview of the Context Management Middleware. Section 7 summarizes the results and provides some insights into future work.

\section{Continuous Care Models}

At present, continuous care models for chronic disease conditions are being discussed, promoted and experimented at national and international level. One of the first examples is the Chronic Care Model (CCM), which is a conceptual, evidence-based 
framework developed in the USA. This model proposes innovative organizational aspects aiming at improving effectiveness and efficacy of assistance to patients affected by chronic conditions. Recently, the WHO has proposed the Innovative Care for chronic conditions (ICCC) framework, which extends CCM for an international community [4].

These models promote an holistic approach for continuous care of chronic conditions, including guidelines for policy planning, as well as principles for social community and health care systems organization and effort coordination.

Focusing on those aspects which are more directly related to the topic of this paper, we highlight that the above-mentioned continuous care models place point-of-care in the community and at patient home. These models emphasize the fact that effectiveness and efficiency of long-term condition care depend strongly on the capability of both patients and their relatives to manage their case (self-management) and on the collaboration of all involved care providers. Patients, family members, health care teams (e.g. clinicians, general practitioner, nurses, etc.) and social community members (e.g., social workers, volunteers) should be properly informed, motivated and prepared in order to effectively collaborate together.

From the point of view of AmI and context-aware system designers, home-based care models pose several technology-oriented challenges. Technology efforts strictly focused on patient assistance requirements provide a relevant but partial contribution in facing the problem. As a matter of fact, collaboration among different stakeholders (health operators, patient relatives, as well as social community members) and efforts coordination requirements should be taken into account in AmI and context-aware system design for continuous care.

\section{Related Work}

There are a number of research projects related to home-care and e-health assistance. Here we will focus on works proposing context-aware systems to support home-based assistance to chronic patients.

Most attempts have focused on specific aspects, such as health status monitoring, alert and reminders based on scheduled activities (e.g., medicine taking, training activities, etc.), patient behavior and daily activities modelling. For instance, Vivago® is an alarm system, providing long-term monitoring of user's activity profile and automatic alarm notification [5]. It consists of three main components: a wrist unit, a base station and an alarm receiving and routing software. Further systems are: Georgia Tech Aware Home, providing assistance services for the elderly [6]; CareMedia [7], which uses multimedia information to track person activities; MIT's PlaceLab [8], which includes a proactive health care application based on wearable and environmental sensing.

The Freeband Awareness project aims at the development of a context-aware infrastructure architecture [9]. The project focuses on requirements coming from the health care domain. Its reference scenarios include tele-monitoring, tele-assistance and assistance to disabled persons.

In above works, guidelines and reference models for continuous care are not yet extensively taken into account in the design of pervasive care solutions. Recently, some high-level analysis and conceptual design have been performed. Chronic care 
model principles have been taken into account in the conception of Pervasive Self Care, a ubiquitous computing service infrastructure conceptual framework [10]. In [2] social network analysis is applied to the study of continuous care networks. First experimentation results have been reported in [11], where a telemedicine system is used for the home care of patients suffering from Chronic Obtrusive Pulmonary Disease; its functionalities include communication facilities between patients and their care team and data sharing among the care team.

While these latter works have focused on conceptual design or on implementation of applications targeted to specific chronic diseases, the aim of this work is to design and implement a service platform supporting care teams which should be dynamically and flexibly activated to provide care services accordingly to patient situation (e.g. health status and home environment).

\section{Application Scenario for Continuous Care at Home}

Novel continuous care models suggest that information and communication systems should provide technological instruments in order to effectively support the activity of continuous care networks and coordinate efforts of different members: patients and families, health care teams and social community partners [4].

In this section we first provide an overview of main stakeholders usually involved in a continuous care network and their requirements, in order to motivate the service platform architecture and the Context Management Middleware for continuous care networks described in the following sections.

With the term continuous care network we refer to the broad range of people which is involved to different extent in the care of the elder. Care networks can be characterized, as specified in [2], in terms of: roles within networks; communication structures within networks; types of information shared; importance of shared information; individuals' comfort in sharing information. Complexity of care network management is also due to the fact that members typically have more than one place of work, belong to different organizations and do not have frequent physical contacts. Availability of shared and updated information is thus a critical aspect for these loosely-coupled teams.

Some relevant stakeholders requirements are described below:

Patient: general patient requirements include the capability of easily communicating with relatives or health operators, notifying an emergency situation and requesting an intervention. He/she might also require support for implementing his/her health assistance plan (for instance, reminding for regular medicine taking and vital sign measurements). Requirements depending on specific patient conditions (e.g. physical impairments, cognitive disabilities, etc.) have not been taken into account in this work.

Family members: providing care can have different impact on patient relatives' life. In [2] three main categories have been identified: those who make drastic life changes to support the elder (e.g. quitting job or neglecting hobbies and social activities); those who make significant contributions to care for the elder (without making important changes on their lives), those who have peripheral involvement in 
the elder's care (e.g. sporadic interaction with the elder). Relatives belonging to the first category are critical network members, as their contribution is relevant for the elder quality of life. As these family members are often involved in implementing the assistance plan, they should be aware of patient conditions, be informed about the health plan implementation (e.g. if the patient has taken his/her medicines) and be made capable of expressing the availability for an intervention (for instance when outside home).

Doctors: Doctors (especially general practitioners) are responsible to define the patient health assistance plan. Accordingly to emerging continuous care models, they should cooperate with nurses and social assistance operators in order to define an integrated assistance plan. Main requirements include the capability of accessing complete and accurate patient records and timely updating patient data (for instance during home visits). Doctors also need not to be overwhelmed by intervention requests, especially when the situation is not extremely critical and could be handled by other operators (nurses, social assistance operators), or when they are involved in critical interventions.

Nurses: They are relevant care providers and, analogously to doctors, they need efficient scheduling management. They also require access to patient records as well as information support for timely interventions.

Social assistance operators: They provide support for daily activities and social communication. Their activity can be relevant also for detecting patient attitudes and emotive state and other information which are useful for evaluating health plan implementation results but could not be automatically measured.

Call center: with this term we refer to the center which has the responsibility of managing incoming calls and alerts and planning interventions. The need is to automate, as possible, the workflow of critical situation management. This roughly consists in detecting critical or emergency situations, deciding which kind of intervention and competences are required, finding available people with needed competences, and contact them via suitable available channels (e.g. email, sms, phone, etc.).

Other community members: community members can be involved to different extent to provide their contribution. They may include volunteers, special transport service associations, shops, chemistries, etc. Their capabilities of service offering can improve the quality of life of both patients and their relatives.

In addition to patient-oriented services (i.e. emergency treatment, autonomy enhancement, and comfort), the design of a context-aware system should also offer services supporting shared care teams. We identified the following services for caregivers: communication facilities; shared access to patient related data; decisionsupport services and automated workflow for critical situation handling and alarm management; mutual "social awareness" about role and action of network members.

Context-awareness is a general concept, referring to the capability of a system to be aware of its physical and logical environment and to intelligently react according to this awareness. Definition of context is thus dependent on application domain and purposes. In our application scenario, context includes information on patient biomedical parameters (e.g. vital signs) and home living environment (for instance air physical parameters such as temperature and relative humidity at patient home). Even the social context (i.e. information regarding persons populating the patient care 
dynamic team) is considered a relevant contextual information to be exploited for context-aware assistance service delivery.

As detailed in the following sections, context management and reasoning services have been conceived and implemented to ease the development of context-aware emergency and autonomy enhancement services. Emergency detection is based on the analysis of vital signs and home environment monitoring. Emergency management is addressed in terms of alert notification planning. These tools can be easily customized for a wide range of patient disease and home living typologies. Support for automatic therapeutic intervention at home is not included since it is a capability implying deep knowledge of the specific patient case and personalized system design.

\section{Context-Aware Service Platform Architecture Design and Deployment}

The overall system architecture is made of the following logical components:

- Context data providers: several physiological sensors are now available which can help patient or relatives for self-measurement (e.g., oxymeter, tensiometer, glycometer, etc.). Activity sensors (accelerometer) and environmental conditions sensors can also be used to enrich system awareness of patient status.

- Context management middleware: this is a distributed architecture of computing nodes (named Context Manager Nodes) devoted to context data acquisition from sensors and analysis for emergency situation detection and handling.

- Context-aware applications: they provide care and assistance services which adapt to the contextual situation (as explained below).

The deployment configuration is hereafter described.

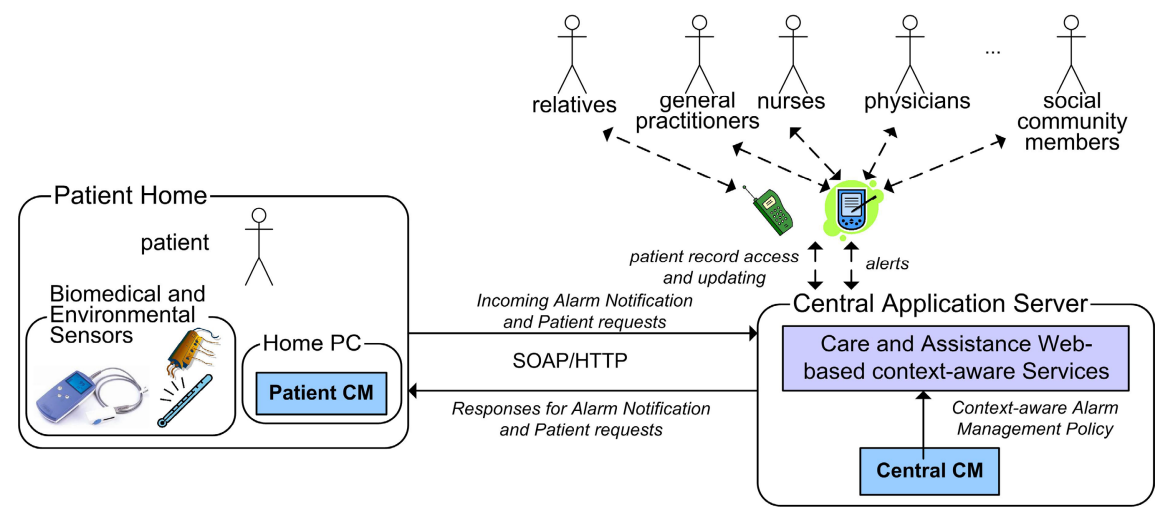

Fig. 1. Service Platform Architecture for Continuous Care

A HomePC (a Tablet PC) is deployed at Patient site. It acquires data coming from biomedical and environmental sensor networks and hosts a node of the Context Management Middleware, named Patient Context Manager (PatientCM). The PatientCM performs context data analysis in order to detect incoming emergency 
situations. It communicates with a Central Application Server via ADSL to send periodical status messages and up-to-date measurement values, as well as to notify alarms triggered by emergency and critical situations.

The Central Application Server is deployed in the domain of the central organization which is responsible for the integration and delivery of care and call center services (for instance, hospitals, nursing homes or municipalities). It hosts the central node of the Context Management Middleware (named Central Context Manager, CentralCM) which works to define an alert notification policy for informing care team members and require their intervention. The Central Application Server is also in charge of delivering assistance and information services.

Main services currently under development include:

- For health care team operators (doctors and nurses): a web-based application which allows team members to access and update patient records, receive alert notifications, manage alarm situations, and set their own availability status for intervention. Through this interface, care providers can notify the system about their interventions, be informed about interventions of other operators and communicate with them. These services can be accessed via desktop PC or PDA.

- For patient relatives: services include access to patient report and capability of notifying the system with their own distance from patient home and receiving alerts via phone call or sms. These services can be accessed via mobile devices with minimal technical requirements (an XHTML browser).

Even if the autonomous access of patients to services and the development of assistive technologies are not the main target of this work, some basic emergency services based on direct patient interaction can be made available by the service platform. For basic testing purposes we are planning the development of a web-based ergonomic Graphical User Interface for medicine assumption reminding and manual alarm and communication requests.

\section{Context Management Middleware Architecture}

This section describes the context management middleware architecture that we have developed to ease the implementation of context-aware assistance services for chronic patients. This system component is based on a general-purpose middleware providing basic features for context data acquisition, reasoning and delivery, which can be easily extended and customized for different application domains [12].

An ontology-based context model is employed throughout the entire process of sensing, interpreting, managing and exchanging context information. We have extended a general purpose context ontology [12] in order to represent contextual situations in a home care setting. Our ontology-based context model for home health monitoring and alerting in patient care networks is described in [13]. Main context modeling and reasoning capabilities are introduced here below.

The context model is composed of the following ontologies:

- the Patient Personal Ontology: includes context items about patient physical data (i.e. biomedical parameters), location and activity. These data are used by the system to automatically infer patient health status and detect alarm situations. 
- the Home Environment ontology: includes context data describing the patient home environment. For instance, monitoring of environment parameters is needed in order to maintain a healthy environment and detect alarm situations.

- The Alarm Management Ontology: represents those resources that could be put in place in order to manage an incoming alarms. This ontology is used in order to instantiate the suitable alarm management policy according to the incoming alarm level, the patient identifier and the availability of care team members.

- The Social Context Ontology: represents care network resources (health teams, social community members, etc.) and their relations.

Context reasoning is used mainly for alarm triggering and management. We have defined a set of first-order rules in order to determine if an alarm has to be triggered and which alarm level should be activated, according to measured context data and corresponding thresholds. The following example shows a rule activating an alarm when both following conditions occur: hearth rate frequency is less than 40 beat/minute and systolic blood pressure is higher than $160 \mathrm{~mm} / \mathrm{Hg}$ :

(?parameter1 rdf:type pre:HeartRateFrequency) (?parameterl pre:hasMeasurementResult ?value1) le(?value1, 40) (?parameter2 rdf:type pre:SystolicBloodPressure) (?parameter2 pre:hasMeasurementResult ?value2) ge(?value 2,160) -> (?healthstatus pre:hasTempAlarmLevel 'HIGH')

Analogously, when the alarm has been activated, rule-based reasoning is used to determine which notification policy should be actuated for alarm handling. An alarm notification policy is a set of instructions specifying whom should be alerted and how (via sms, mail, phone call) and if acknowledge is required.

The Kamer Context management middleware is composed of context management nodes, named Context Managers (CM) and its operation is based on the above mentioned context modeling and reasoning principles. The logical architecture is similar for both the Patient Context Manager and the Central Context Manager (see Fig. 2). The Context Data Acquisition module receives context data from context providers via SOAP messages (e.g. web service-enabled sensor networks [14]), or via sensor adapters. The Ontology Manager manages the knowledge base, composed of context models and instances stored in a database, a rule-based reasoner and rule files. A Context Broker component makes context information available to external applications, by managing queries from applications and/or notifying interested applications when the context has changed. Communication among distributed components is provided via SOAP Web Services.

Specifically, the Patient Context Manager receives context data from body sensor networks and home sensors. These data are used to update the Patient Personal and the Home Environment Ontologies instances. Rule-based reasoning is applied to the knowledge base in order to detect possible alarms. If an alarm occurs, the PatientCM notifies the Central CM. Then, the CentralCM applies rule-based reasoning to the knowledge base composed by the Social Context Ontology (containing updated information about patient relatives and care providers availability) and the Alarm Management Ontology (instantiated with the information about the alarm situation to be handled). The resulting alarm notification policy is then instantiated by the Alarm Notification Application. 


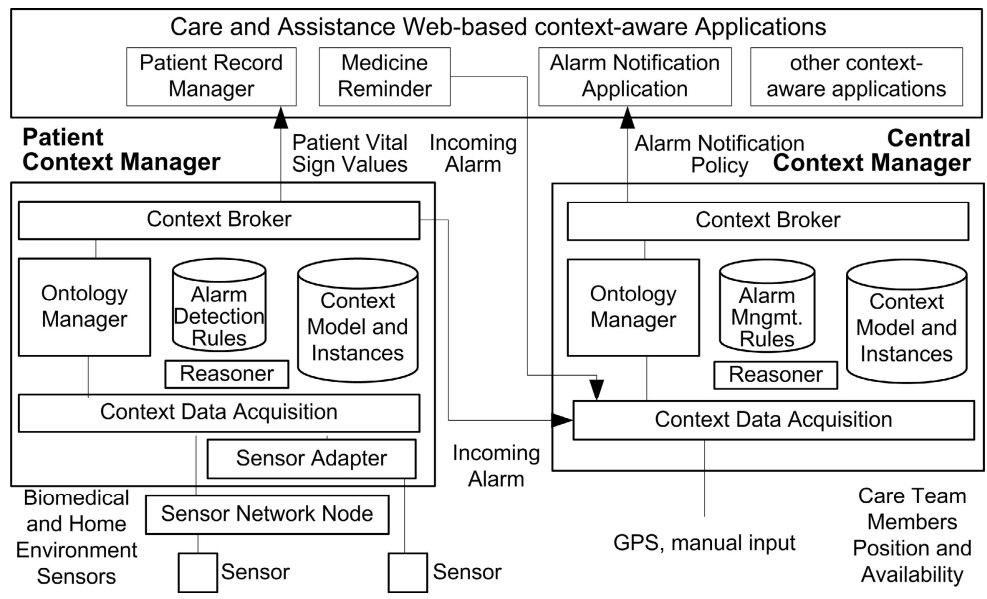

Fig. 2. Context Management Middleware Architecture

The Context Manager has been implemented as a J2EE application. Context Model and instances are encoded in OWL (Web Ontology Language) [15] and stored in a relational database (MySQL). The Ontology Manager and the reasoner are based on Jena [16], a Java framework for building Semantic Web applications.

\section{Conclusions}

In this paper, we have presented a context-aware service platform designed for homebased assistance of chronic patients. Main distinguishing features of this work are: the attempt to systematically take into account requirements coming from novel continuous care models into system design; the design of a service platform for continuous care networks leveraging on a Context Management Middleware and an ontology-based context model for health monitoring and alert management.

The Context Management Middleware has been described with some design details. Services for patient assistance and care network support are currently under development. Use-case testing is planned with a trial in a nursing home in the near future. This testing stage will be focused on evaluating health operators acceptance of implemented features. For this test stage, biomedical and environmental sensing will be simulated by a web application providing services for manual input or pre-defined scenario simulation for context data acquisition. A testing stage with chronic condition patients will be planned in the near future. For these tests, a sensing system for vital signs and environmental monitoring will be deployed. Test results will be reported in the conference presentation.

Acknowledgements. The Kamer Project is funded by the Regione Emilia Romagna, Italy. The partners are Hewlett Packard Italy, Cattolica University of Piacenza and the Italian National Inter-University Consortium for Telecommunications (CNIT). 


\section{References}

1. Börsch-Supan, A., Jürges, H.: The Survey of Health, Ageing and Retirement in Europe Methodology. Mannheim Research Institute for the Economics of Aging, MEA, Mannheim (2005)

2. Consolvo, S., Roessler, P., Shelton, B.E., LaMarca, A., Schilit, B., Bly, S.: Technology for Care Networks of Elders. IEEE Pervasive Computing 3(2), 22-29 (2004)

3. Nehmer, J., Karshmer, A., Becker, M., Lamm, R.: Living Assistance Systems - An Ambient Intelligence Approach. Proceedings of the 28th International Conference on Software Engineering (ICSE 2006), Shanghai, China (2006)

4. World Health Organization (WHO): Innovative Care for Chronic Conditions: Building Blocks for Action ( 2002), http://www.who.int

5. Korhonen, I., Paavilainen, P., Särela, A.: Application of ubiquitous computing technologies for support of independent living of the elderly in real life settings. Proc. UbiHealth 2003, The 2nd International Workshop on Ubiquituos Computing for Pervasive Healthcare Applications (2003)

6. Abowd, G.D., Bobick, I., Essa, I., Mynatt, E., Rogers, W.: The Aware Home: Developing Technologies for Successful Aging. Proc. of American Association of Artificial Intelligence (AAAI) Conference (2002)

7. Bharucha, A.J.: CareMedia: Automated Video and Sensor Analysis for Geriatric Care. Presented at the Geriatric Care Annual Meeting of the American Association for Geriatric Psychiatry, San Juan, Puerto Rico (2006)

8. Intille, S.S., Larson, K., Tapia, E.M.: Designing and evaluating technology for independent aging in the home. Proc. of the International Conference on Aging, Disability and Independence (2003)

9. van Kranenburg, H., Bargh, M.S., Iacob, S., Peddemors, A.: A context management framework for supporting context-aware distributed applications. IEEE Communications Magazine 44(8), 67-74 (2006)

10. Roussos, G., Marsh, A.: A Blueprint for Pervasive Self-Care Infrastructures. Proc. of Fourth International Conference on Pervasive Computing and Communications, Workshop on Ubiquitous and Pervasive HealthCare (2006)

11. De Toledo, P., Jimenez, S., Del Pozo, F., Roca, J., Alonso, A., Hernandez, C.: A telemedicine experience for chronic care in COPD. IEEE Transactions on Information Technology in Biomedicine 10(3), 567-573 (2006)

12. Paganelli, F., Bianchi, G., Giuli, D.: A Context Model for Context-aware System Design towards the Ambient Intelligence Vision: Experiences in the eTourism Domain. Proc. of 9th ERCIM Workshop User Interfaces For All, Special Theme: Universal Access in Ambient Intelligence Environments, Königswinter (Bonn), Germany (2006)

13. Paganelli, F., Giuli, D.: An Ontology-based Context Model for Home Health Monitoring and Alerting in Chronic Patient Care Networks. Accepted at First International Workshop on Smart Homes for Tele-Health, AINA (2007)

14. Woo, A.: A New Embedded Web Services Approach to Wireless Sensor Networks. Proc. of the 4th International Conference on Embedded Networked Sensor Systems (2006)

15. Web Ontology Language, W3C recommendation. http://www.w3.org/TR/owl-guide/

16. JENA: A Semantic Web Framework for Java [Online]. http://jena.sourceforge.net/ 